\title{
Profiling of aerosol concentrations, particle size distributions and relative humidity in the atmospheric surface layer over the North Sea
}

\author{
By GERRIT DE LEEUW, Physics and Electronics Laboratory TNO, P.O. Box 96864, \\ 2509 JG The Hague, The Netherlands
}

(Manuscript received 21 November 1989; in final form 2 February 1990)

\begin{abstract}
Results are presented from measurements in the lower $15 \mathrm{~m}$ of the marine atmospheric surface layer, of sea spray particle size distributions, intensities of light scattered by these particles in the near-forward direction, and relative humidity. Particle size distributions were measured with a Rotorod rotating impaction sampler. Variations of particle total area concentrations were determined from measurements of forward scattering intensities with an angular scattering device (further referred to as an optical scatterometer). This latter instrument was used to measure both time-dependent variations and mean values, at fixed heights between 1 and $15 \mathrm{~m}$ above the mean sea surface and wave-following at heights between 0.5 and $3 \mathrm{~m}$. The paper is focussed on the comparison of the profile data obtained with the Rotorod and the optical scatterometer. These instruments are based on different physical principles. The shapes of the particle forward-scattering profiles are in good agreement with the particle concentration profiles measured with the Rotorod, provided that ambient conditions do not change during the consecutive measurements. The observed trends in the particle profiles and in the humidity profiles are also similar, reflecting the interaction between the relative humidity and sea-spray droplets. Results from the time-dependent measurements, which have been published elsewhere, are briefly summarized in the context of the analysis of the profile measurements. They show that fluctuations of particle total area concentrations are correlated with the motion of the waves under the sampler. Simultaneous time-series measurements of relative humidity do not reveal a distinct wave influence. This suggests that water vapour is transported by turbulent (molecular) diffusion on a time scale that is much faster than the time scale for particle transport. This is due to the inertia of the largest particles, which respond much slower to changes in the air flow than passive molecular tracers. These results are significant in the context of humidity transport and the constant flux assumption in the surface layer. They are also important for the insights they may provide as to flow interaction with the waves in the open ocean, using the aerosol as a tracer. This requires a comprehensive theoretical analysis which is outside the scope of this paper.
\end{abstract}

\section{Introduction}

Particle-mediated transport of liquid water may contribute substantially to the humidity and heat exchange over the sea. A significant increase in the moisture content of the surface layer may be caused by the evaporation of freshly produced sea-spray droplets, but also liquid-vapour conversion at higher altitudes may affect the humidity profile. Little information is available, however, both on the production of particles at the sea surface and on the subsequent dispersal. The production estimates by Monahan et al. (1986) rely on an empirical model based on whitecap observations combined with wave tank measurements of the production rate per whitecap area. Fairall et al. (1983), on the other hand, derived their estimates from application of a mixed-layer model to particle size distributions measured from a ship at a height of about $10 \mathrm{~m}$. As shown 

elsewhere (De Leeuw, 1989a), the vertical variation of particle concentrations throughout the boundary layer as predicted by this model compares favourably with experimental data obtained with lidar. This, combined with the good agreement of the mixed-layer model with observed aerosol concentrations at $10-\mathrm{m}$ levels (Fairall et al., 1984) suggests that the used surface-layer profile relationship yields a useful estimate for the effective value of the flux at $10 \mathrm{~m}$. This does not imply, however, that the flux right at the surface is also correct. To verify this, comparisons have been made between measured surface-layer aerosol profiles and profiles calculated with the mixed-layer model. In the surface layer good agreement is often obtained for particles smaller than about $15-20 \mu \mathrm{m}$, but only at low-wind conditions when whitecapping is insignificant. For larger particles that are most important for humidity transport, the model fails to predict the correct shapes of the profiles, while at moderate to high winds the model also fails for the 15-20 $\mu \mathrm{m}$ particles (De Leeuw, 1988). Obviously, the surface-layer flux profile relationship that is used in the model is not correct very close to the surface. As a consequence, using the mixed-layer model for extrapolation of aerosol concentrations at $10 \mathrm{~m}$ to estimate the surface production rate may yield erroneous results, in particular for the larger particles.

Experimental evidence exists that at high winds the particle concentration profiles between 0.2 and $20 \mathrm{~m}$ above the sea surface do not vary exponentially with height all the way down to the surface (De Leeuw, 1986a, 1987). Minima and maxima have been observed in the concentration profiles which become more obvious as wind speed increases. The lowest wind speed at which this has been observed is $7 \mathrm{~m} / \mathrm{s}$. These results were explained in terms of a wave-rotor model, see Fig. 1. In the proposed model an eddy airflow is assumed to come into existence in the wave troughs under breaking wave conditions. The particles are assumed to be temporarily trapped in the eddies. Hence, the action of the wave rotor results in longer suspension times, which could explain the shape of the profiles. This has been discussed in more detail in De Leeuw (1986a, 1987). If the particle suspension time is enhanced by trapping of the particles in the eddy air flow, more time would be available for evaporation

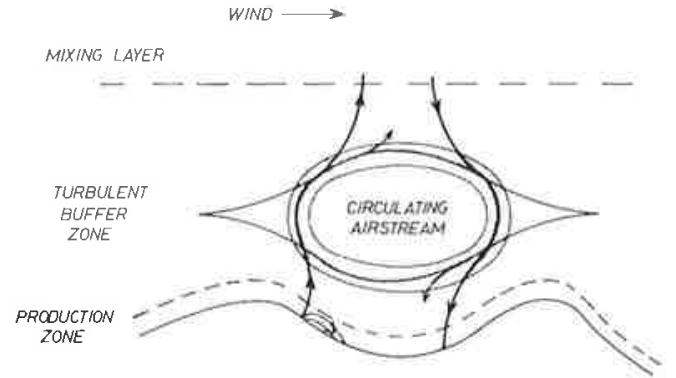

Fig. 1. Schematic representation of the wave-rotor model.

which affects the moisture content of the surface layer and its latent heat flux.

The occurrence of flow separation has only been observed in laboratory experiments (e.g., Hsu et al., 1981; Banner and Melville, 1976; Konishi, 1981), and in one field experiment over a lake (Anisimova et al., 1976). No data exist which indicate the occurrence of flow separation in the open ocean where the waves are much longer and higher, probably because it is extremely difficult to obtain such data in open ocean conditions. It should also be realized that a variety of processes may influence the aerosol concentrations near the air-sea interface. Both the production mechanism and the interaction of the droplets with the turbulent fields of air flow and humidity should be considered. Turbulence is the dominant mechanism that keeps the particles in suspension in the atmosphere, i.e, it enhances the residence time (with respect to still air). Thus the particles can adjust to relative humidity by evaporation and condensation. In this respect there is no difference between enhanced suspension times due to the proposed wave-rotor mechanism or due to other manifestations of turbulence. There is no reason, however, why extended suspension caused by turbulence would result in the observed particle-concentration profiles, with a maximum at heights of $1-2 \mathrm{~m}$. Turbulence is expected to result in logarithmic profiles, as deduced from surface-layer similarity theory (Fairall and Davidson, 1986).

The two different production mechanisms, viz. bubble-mediated production of film and jet drops and direct production by wave disruption (see Monahan et al., 1986), may affect the profile as well. The two production processes take place at different heights with respect to the water 
surface. Film and jet drops are generally produced in the wave troughs, while direct production occurs at the crests. The latter becomes more important as wind speed increases and may thus contribute to the maximum in the aerosol profile that develops at higher wind speeds at levels near the wave crests. This production mechanism at elevated heights cannot explain the second maximum described in De Leeuw (1987), however. Also, the observed wind-speed dependence of the particle concentrations does not indicate different wind-speed regimes. Different wind-speed regimes would exist if a supplementary source would come into existence, such as spume droplet production at wind speeds greater than 9-11 m/s (Monahan et al., 1986).

Another point to be considered in this respect is the ejection height of the jet droplets. Laboratory observations of the ejection of jet droplets in still air by single bubbles indicate a maximum ejection height of $18 \mathrm{~cm}$ (e.g., Blanchard, 1983). In a bubble plume, on the other hand, these ejection heights may be extended to higher levels (Mestayer and Lefauconnier, 1988). In the field these ejection heights may be higher due to differences in both the intensity and the scale of the turbulence, giving the droplets an extra lift in addition to their initial ejection velocity. Also surface displacements need to be considered because the waves cause a constantly varying surface level during the ascent and fall of the droplets.

Open ocean observations by Bortkovskii (1987) indicate the top of the trajectory of freshly produced droplets at approximately the "thickness of the frontal part of the breaker, about one meter, under storm conditions". Ejection heights of one meter would result in the observation of a maximum in the mean concentrations at that height because the probability to detect a "lingering" particle is much higher than the probability to detect a transient particle.

Aerosol production is restricted to limited seasurface areas: those where bubbles, produced by whitecapping or biological activity, reach the surface (whitecaps cover at best a few percent of the sea surface, although the "invisible" bubble plume consisting of smaller bubbles, exists appreciably longer (Monahan, 1988); thus, the production spectrum will also change with the age of the whitecap-induced bubble plume); and, at high wind speeds (larger than about $9 \mathrm{~m} / \mathrm{s}$ ), to the wave crests where droplets are produced by wave disruption.

Thus, production is not continuous over the wave field. However, due to vertical mixing and horizontal advection over many waves, the discontinuous production has no significant effect on the observed concentrations, except maybe very close to surface for the largest particles which fall immediately back into the sea. In our measurements with the Rotorod the data are averaged over four minutes, i.e., typically over $50-100$ waves in the North Sea.

The discontinuous production of aerosol is confirmed by the experiments by $\mathrm{Wu}$ et al. (1984), who observed bursts of droplets, larger than $40 \mu \mathrm{m}$ in diameter, occurring at low elevations at rather regular intervals at wind speeds of $6-8 \mathrm{~m} / \mathrm{s}$. The fluctuating droplet concentrations are correlated with the wave phase.

Laboratory experiments at wind speeds of $16 \mathrm{~m} / \mathrm{s}$ by Koga and Toba (1981) show that the highest particle concentrations occur in the lee of the wave. The observed particles were produced in the same wave trough at the downwind slope. However, as indicated by De Leeuw (1989b), in the field the production may be higher on the windward side of the crest.

On the other hand, when production occurs over limited sea surface areas, the remainder of the sea surface serves only as a sink. Likely, the concentrations are lower in these "sink regions" than in the "source regions". Averaging over the whole sea surface might yield the apparently unphysical result that the concentration is higher at some elevated level than right at the surface.

The interaction with the waves should also be considered. Deposition of the particles on the water surface due to gravitational and turbulent processes, further influenced by differences in flow velocities and wave phase velocity, leads to the reduction of the aerosol concentrations in the wave troughs. If the aerosol in the troughs is not replenished by freshly produced droplets, this leads to concentrations that are lower than just above the wave tops. Consequently, wave-following sampling of mean aerosol concentrations may result in lower concentrations as the sampler spends more of the total sampling time in the troughs. This occurs when the sampler is mounted on the wave-follower at levels which are 
lower than the wave height. As a result, the measured concentrations might decrease with height from the wave tops down to the surface. This suggests that our previous observations in the North Atlantic (De Leeuw, 1986a) could be the result of the sampling method used. Observations over the North Sea, however, indicate the occurrence of a second maximum which cannot be explained this way. As discussed in De Leeuw (1987), this second maximum is a real phenomenon that is not caused by the transition from sampling on a wave-follower to sampling at fixed height.

In the above, processes have been indicated which may influence the aerosol profile in the marine surface layer. Until a comprehensive numerical model has been developed which describes these processes and shows which prevail and which are less important or can even be neglected under various atmospheric conditions, it is all speculation. For the development of such a model quantitative information is required. Measurements of particle size distribution profiles alone do not yield conclusive information on the relative importance of the above processes of production and dispersal. Therefore, during the humidity exchange over the sea (HEXOS) main experiment HEXMAX (see Katsaros et al. (1987) for an overview) the temporal variations in the particle concentrations were also measured. This was achieved with an optical device (further referred to as an optical scatterometer) that measures particle total area concentrations from the intensity of near-infrared laser light that is scattered by the particles in the sample volume between angles of $15^{\circ}$ and $35^{\circ}$ from the forward direction. The sample frequency was $200 \mathrm{~Hz}$ in the used configuration. The optical scatterometer was mounted on our wave-rider buoy, together with a capacitance hygrometer $(\max$. response rate $1 \mathrm{~Hz}$ ), to determine fluctuations of particle total area concentrations and relative humidity at heights between $0.5 \mathrm{~m}$ and $3 \mathrm{~m}$ above the instantaneous sea surface. The profiles were completed with measurements at fixed height above the mean sea surface between 1 and $15 \mathrm{~m}$. Using a mast, samples were taken at $13 \mathrm{~m}$ from the platform structure to reduce flow distortion. In addition to the measurements with the optical scatterometer and the hygrometer, particle size distribution profiles were determined with Rotorod rotating impaction samplers (cf. De Leeuw, 1986a, b). These efforts were particularly focussed on the lower $5 \mathrm{~m}$.

The HEXMAX experiment was carried out in October and November 1986 from Meetpost Noordwijk, a tower in the North Sea at $9 \mathrm{~km}$ from the coast of the Netherlands. The objectives of our measurements during the HEXMAX experiment were as follows.

(1) To confirm previous observations of the variation of particle concentration profiles in the surface layer (De Leeuw, 1986a, 1987). Therefore these profiles were measured both with Rotorod impaction samplers and with the optical scatterometer. These measurements are based on different physical principles (impaction and scattering). Thus we have two independent methods. The Rotorod measurements and those with the optical scatterometer were made consecutively. Physical limitations made it impossible to perform the measurements concurrently.

(2) To test the hypotheses involved in the wave-rotor model that was proposed to explain the previous observations as discussed above. Therefore not only mean particle properties were measured, but also the temporal variations of the scattering properties.

(3) To obtain experimental information on the various production and dispersal processes near the air-sea interface and the interaction with the turbulent fields of air flow, humidity and temperature. To achieve this goal a theoretical analysis is required which is outside the scope of this paper.

The experiments are described in Sections 2-5. Results from the height- and time-dependent measurements are presented in Sections 6-9.

The paper focuses on the comparison of profiles of particle size distributions as measured with the Rotorod, profiles of particle total area concentrations as measured with the optical scatterometer, and humidity profiles (objective 1 ), see Section 7. The time-dependent measurements have been discussed in De Leeuw (1989b). Another example of the wave-induced temporal fluctuations of the total area concentrations, and its associated frequency spectrum, is presented in Section 8. The results of the time-dependent measurements are summarized in Sections 8 and 
9 , in relation to the comparison of the various profiles. A discussion follows in Section 10.

\section{Measurements of particle size distributions}

Size distributions of particles larger than $10 \mu \mathrm{m}$ in diameter were measured with a Rotorod inertial impactor. The sampler consists of two polished stainless steel rods, mounted in a retracting collector head on a motor which rotates at a nominal speed of $2400 \mathrm{rpm}$. The linear velocity of the rods is $10 \mathrm{~m} / \mathrm{s}$. Particles impacted on the rods are retained by a sticky coating (silicone). Microscope images of the rods are digitized to determine the particle size distribution by computer. The performance of the Rotorod was discussed in a previous paper (De Leeuw, 1986b), where also a comparison was made between marine aerosol spectra measured with the Rotorod and with other instruments.

\section{Measurements with the optical scatter- ometer}

Particle total area concentrations were measured from the intensity of laser light scattered by droplets in the open sample volume of an optical scatterometer. The instrument was especially constructed for the HEXMAX experiment. It is shown schematically in Fig. 2. In brief, pulsed near-infrared radiation emitted by a diode laser (wavelength $0.904 \mu \mathrm{m}$ ) is guided by an optical fiber to the emitter side. The divergent laser beam exciting the fiber is collimated with a lens to form a parallel beam that is directed into the sample volume, making an angle of $25^{\circ}$ with the receiver optical axis. The receiver consists of



Fig. 2. Schematic representation of the optical scatterometer. a photodiode and a lens and has a field of view of $20^{\circ}$. In this configuration radiation is detected that is scattered between angles of $15^{\circ}$ and $35^{\circ}$ from the laser beam. The detected pulses are amplified and further processed with a synchronized peak detector and a computer-controlled analog to digital converter. The data recording speed was about $200 \mathrm{~Hz}$ in this configuration. The response of the optical scatterometer is proportional to the aerosol total area concentration.

The emitter laser diode and the receiver photodiode are mounted in a single water-tight box which also contains the electronics. The emitter end of the optical fiber, and the associated lens and housing, are connected by two rigid rods to the water-tight box, allowing a free airflow through the sample volume. The receiver looks into a beam dump to shield it from direct solar irradiation and reflections from the water surface which may cause spurious background signals. Both units are mounted in protective cylinders to shield them from the optical degradation associated with the turbulent deposition of sea spray aerosols on windows and lenses. The optical scatterometer weighs only $2.4 \mathrm{~kg}$, exclusive of the cables for power supply and data transmission. This light weight allows for the mounting of the instrument on a simple wave-following buoy.

The optical scatterometer was designed as an instrument with a fast response for quickly profiling of the aerosol in the atmospheric surface layer. To achieve this, the signals at distinct levels were integrated to obtain mean values. A second application of the instrument is the recording of time series to obtain information on temporal behaviour of aerosol properties.

\section{Humidity measurements}

Relative humidity was measured with a capacitance hygrometer (VAISALA). The response time of this instrument is about $1 \mathrm{~s}$. This hygrometer was mounted in a $30 \mathrm{~cm}$ long and $10 \mathrm{~cm}$ wide protective tube with forced aspiration, to shield the sensitive element from contamination by sea-spray droplets. This shielding increased the life time of the sensitive element appreciably. In the open configuration (i.e., unshielded by the extra protective tube, but with the small protective cap delivered by the manu- 
facturer), sea salt was observed on the sensitive element after almost every profile. In the shielded configuration this occurred only rarely. The aspirator consisted of a fan powered by a $12 \mathrm{~V}$ battery package. Energy consumption, and heat production, were low so that the temperature near the sampler was not affected.

The sensitive element was cleaned or replaced whenever degradation by salt contamination was observed. After each measurement the hygrometer was calibrated versus other humidity sensors at the MPN.

\section{Experiments}

Particle size distributions, particle total area concentrations and relative humidities were recorded at heights from some tenths of meters above the water surface up to $15 \mathrm{~m}$, at a distance of $13 \mathrm{~m}$ from the platform. This was achieved by using a horizontal mast extending North from the NW corner of the platform. As deduced from data presented in Wills (1984), flow perturbation due to the platform is negligible at this position. Also the influence of waves breaking on, or reflected at, the platform structure was not noticeable for the relevant wind directions.

For wave-following measurements the samplers were attached to a float, see Fig. 3. The lowest levels above the instantaneous water surface at which the particle size distributions were measured from the float were $0.12 \mathrm{~m}$ in calm seas, and $0.35-0.5 \mathrm{~m}$ in rough water. For the measurements with the optical scatterometer and the hygrometer, the lowest level was $0.5 \mathrm{~m}$ above the instantaneous sea surface. These wave-following measurements were made to a highest level of $3 \mathrm{~m}$ above the instantaneous sea surface and the distances between successive levels were $0.25 \mathrm{~m}$. Above this height, data were recorded at fixed heights above the mean sea surface, with a separation of $1 \mathrm{~m}$ between successive levels.

Each profile was constructed from two series of measurements: one starting at the top going down toward the sea surface in discrete steps of twice the vertical resolution, and another profile starting near the surface going up in discrete steps while filling up the gaps in the downward profile. This procedure was followed to be able to detect errors due to pollution by sea spray on the

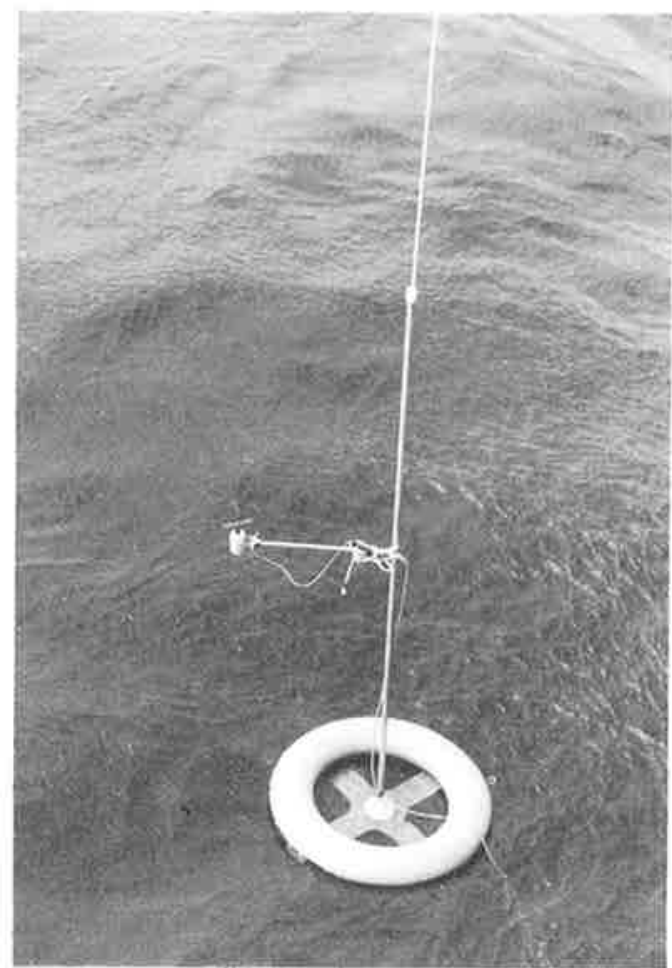

Fig. 3. Waverider buoy with Rotorod impaction sampler.

windows of the optical scatterometer or on the humidity sensor element. Pollution by a sea spray droplet could clearly be observed from the differences between the up-going and down-going profiles. Whenever this was observed, the results were rejected for further analysis.

\section{Particle size distributions}

Particle size distributions measured with the Rotorod were presented in previous publications (De Leeuw, 1986a, 1987). Comparisons with particle size distributions measured with other methods were presented in De Leeuw (1986b). These earlier data were recorded at a maximum wind speed of $13 \mathrm{~m} / \mathrm{s}$. During the HEXMAX experiment the wind speed often exceeded this value. In the storm on 20 October 1986, some samples were taken from the deck of the platform at a height of about $11 \mathrm{~m}$ above the mean sea 


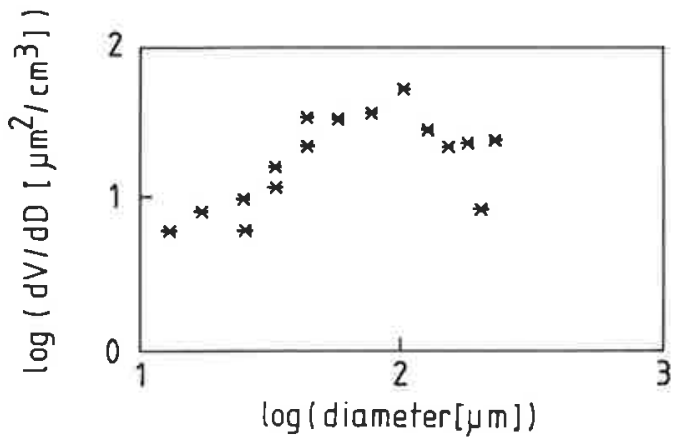

Fig. 4. Particle volume distribution measured with the Rotorod impaction sampler in the storm of 20 October 1986 , from the deck of the platform at a height of about $11 \mathrm{~m}$. The wind speed was $25 \mathrm{~m} / \mathrm{s}$. Note the mode at $100 \mu \mathrm{m}$. Systematic errors are 10-20\%, statistical sampling errors are approx. $10 \%$ for particles smaller than $75 \mu \mathrm{m}, 14 \%$ for $100 \mu \mathrm{m}, 27 \%$ for $125 \mu \mathrm{m}, 41 \%$ for $150 \mu \mathrm{m}, 50 \%$ for $175 \mu \mathrm{m}$, and $70 \%$ for $200 \mu \mathrm{m}$.

surface (unfortunately profiles could not be measured at these high wind speeds). The particle volume distribution measured at $13.25 \mathrm{GMT}$, at a wind speed of $25 \mathrm{~m} / \mathrm{s}$, is presented in Fig. 4. The wave field was not fully developed during the storm. The particle size distribution has an extreme shape with a strong mode near $100 \mu \mathrm{m}$.
In the evening the wind went down to $20 \mathrm{~m} / \mathrm{s}$. The shape of the particle size distributions hardly changed, however, for particles up to $75 \mu \mathrm{m}$. The volume concentrations of larger particles decreased slightly, resulting in a shift of the maximum volume concentration to $75 \mu \mathrm{m}$. This particle size distribution persisted all evening.

\section{Profiles}

Examples of mean profiles of particle total area concentrations, humidities and particle size distributions are presented in Figs. 5-7. The data from the optical scatterometer and the Rotorod are often similar (see however the discussion below on the discrepancy between the particle data in Fig. 5) and in agreement with our previous observations on the shape of particle size distribution profiles (De Leeuw, 1986a, 1987). In many cases minima and maxima were observed in a rather regular pattern. At low wind speeds the concentrations increase toward the surface (e.g., the optical scatterometer data in Fig. 5), but not as fast as would be expected from the theory (cf., the discussion in the introduction). At higher wind speeds a maximum may develop (cf., Fig. 6) which is conjectured to be caused by the action of
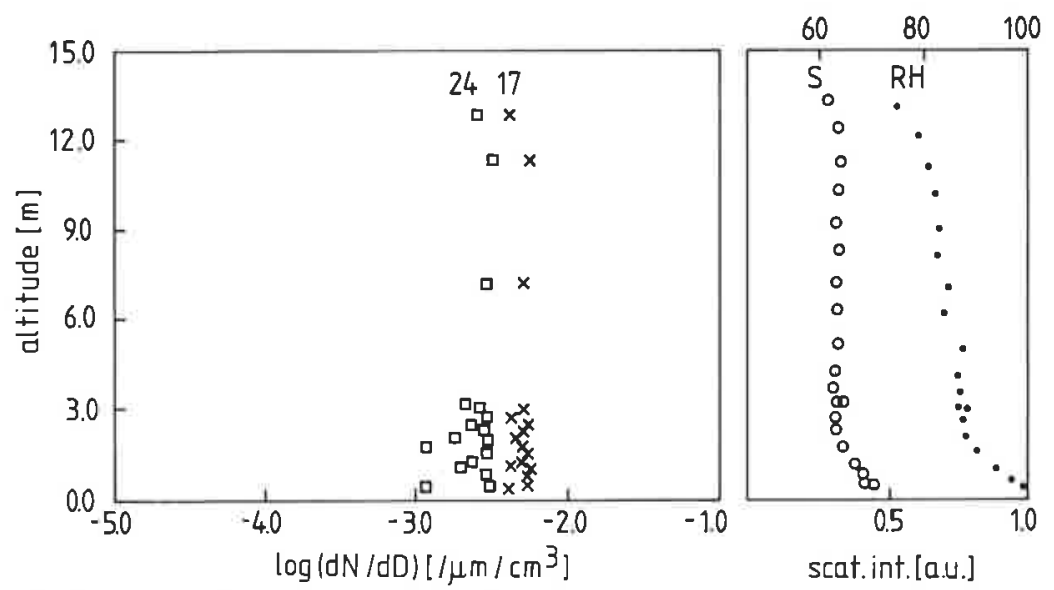

Fig. 5. Profiles of particle size distributions (l.h.s. figure), mean particle total area concentrations measured with the optical scatterometer (S) and relative humidity (RH) measured on 15 November 1986, at a wind speed of $7.7 \mathrm{~m} / \mathrm{s}$. The numbers on top of the profiles in the $1 . \mathrm{h} . \mathrm{s}$. figure indicate the particle diameters. The signal of the optical scatterometer is given in arbitrary units [A.U.] because the instrument had not been calibrated for these experiments. Note the strong gradients near the surface in both the particle total area concentrations and the relative humidity. Systematic errors in the Rotorod measurements are 10-20\%, statistical errors are approx. $10 \%$. The scattering intensities are averages over 1000 samples, relative humidities are averaged over 50 samples. Profiles were measured as described in the text. 

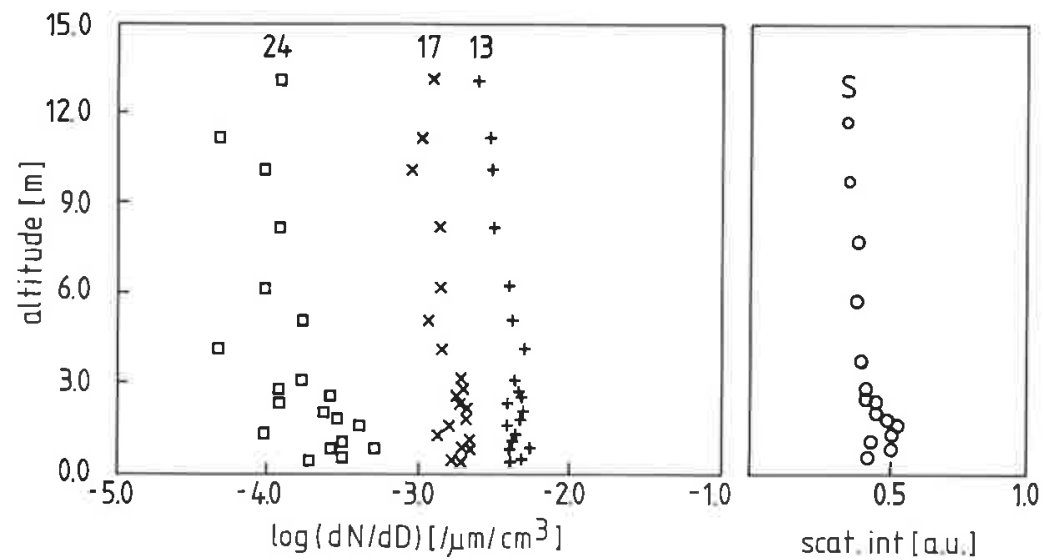

Fig. 6. As Fig. 5, measured on 22 October 1986, at a wind speed of $12 \mathrm{~m} / \mathrm{s}$. Humidity data are missing because of malfunctioning of the hygrometer. Statistical errors in the Rotorod data are approx. $14 \%$ for particles of $13 \mu \mathrm{m}$, $20 \%$ for $17 \mu \mathrm{m}$, and $35-70 \%$ for $24 \mu \mathrm{m}$.

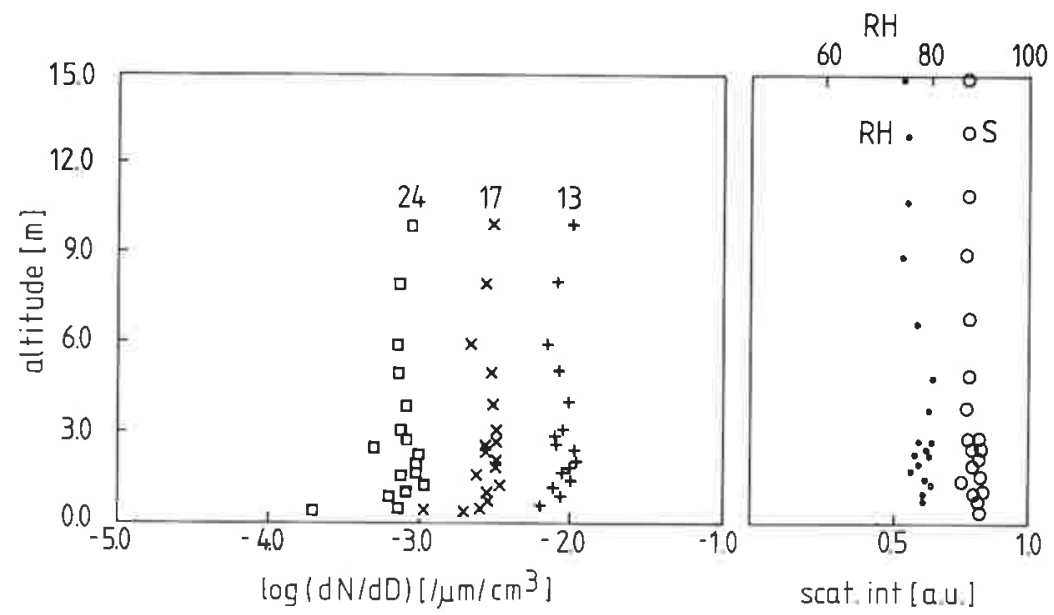

Fig. 7. As Fig. 5, measured on 24 October 1986, at a wind speed of $14 \mathrm{~m} / \mathrm{s}$. Statistical errors in the Rotorod data are approx. $5 \%$ for particles of $13 \mu \mathrm{m}, 9 \%$ for $17 \mu \mathrm{m}$, and $25 \%$ for $24 \mu \mathrm{m}$.

the wave-rotor mechanism. In other high wind cases, however, particle concentrations were observed that were practically independent of height (Fig. 7). The features are size-dependent, which is clearly demonstrated in the Rotorod data.

In some cases, discrepancies were observed between the particle concentration profiles determined with the optical scatterometer and the particle size distribution profiles that were measured with the Rotorod. Fig. 5 is an example of this discrepancy, which may have two causes. In the first place, the optical scatterometer data were averaged over about $50 \mathrm{~s}$ while the particle size distribution profiles are averages over $4 \mathrm{~min}$. As shown in De Leeuw (1989b), see also Fig. 8, the particle concentrations are subject to fluctuations caused by the surface displacements. Therefore averaging over many wave periods is required to obtain reliable mean values.

A more likely cause for the discrepancy between some of the profiles, such as those in Fig. 5, is the separation in time of the measurements with the optical scatterometer and with the impaction method, which in some cases was 1-2 $\mathrm{h}$. During this time the ambient conditions 


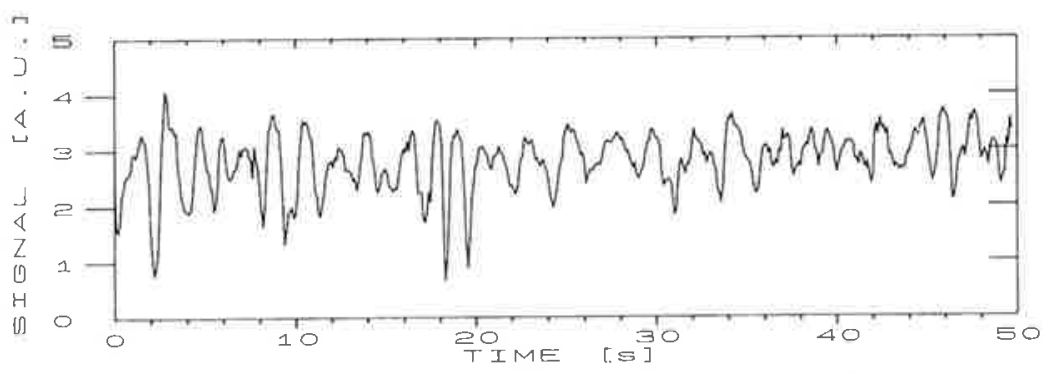

Fig. 8. Time-dependent fluctuations of particle total area concentrations of A.U. (arbitrary units), measured at $2.5 \mathrm{~m}$ above the instantaneous sea surface with the optical scatterometer mounted on a waverider buoy. Wind speed was $12.1 \mathrm{~m} / \mathrm{s}$. The variations in the signal of the optical scatterometer are correlated with the surface displacements. The standard deviation in the data is approx. $20 \%$.

could have changed appreciably. This in fact happened during the measurements in the afternoon of 15 November, which might explain the difference in Fig. 5 between the profiles that were measured with the optical scatterometer and those determined with the Rotorod. The particle size distribution profiles were measured in the afternoon, just before sunset. The data near the surface show some scatter, but there is no obvious height dependence. The particle total area concentration profiles and the humidity profiles, on the other hand, show a strong gradient near the air-sea interface, which was reproduced in several subsequent measurements during the same evening. These profiles were measured after sunset and ambient conditions had changed. Ambient relative humidity (measured at $16 \mathrm{~m}$ above sea level) had decreased from $90 \%$ to $75 \%$, the mixed-layer height was decreased after sunset, as expected and confirmed by lidar measurements. The surface backscatter coefficients were increased, indicating an increase in the particle concentrations caused by the decrease of the mixed-layer depth. The consecutive measurements with the optical scatterometer confirm the increase in the particle concentrations, which is further supported by the particle size distributions measured with optical particle counters from the deck of the platform.

The data in Figs. 6 and 7 are examples of good agreement between the two particle sampling methods. In Fig. 6, profiles are presented that were measured at a wind speed of $12 \mathrm{~m} / \mathrm{s}$. A maximum is clearly observed between 1 and $2 \mathrm{~m}$. In the profiles presented in Fig. 7, on the other hand, the particle concentrations are practically independent of height. The latter data were recorded at a wind speed of $14 \mathrm{~m} / \mathrm{s}$. The reason for the difference between the shapes of the profiles in Figs. 6 and 7 is not clear. At this moment one can only speculate about the causes. Possibly the ambient conditions during the measurement of the profiles in Fig. 6 were more in favour of the wave-rotor mechanism, whereas the meteorological conditions during sampling of the data presented in Fig. 7 were more in favour of turbulent transport. Further analysis, following the theoretical approach outlined in De Leeuw $(1989 \mathrm{~b})$, see also the discussion in the introduction of this paper, is expected to yield better insight into the processes that determine particle transport near the sea surface.

Similarities are observed between the shape of the humidity profiles and the simultaneously measured profiles of particle total area concentrations, see Figs. 5 and 7. Both profiles show a strong increase toward the surface in Fig. 5, whereas in Fig. 7 both profiles show little height dependence. In Fig. 7, a decrease $(\sim 5 \%)$ is observed in the humidity profile between 5 and $7 \mathrm{~m}$, without a corresponding feature in the aerosol profile.

The similarity between the humidity and aerosol profiles is caused by the interaction between the relative humidity and the hygroscopic sea-salt aerosols. Near the sea surface freshly produced particles, with the composition of sea water, evaporate until equilibrium is reached with the ambient relative humidity. Obviously this enhances the local water vapour concentration. The effect of droplet evaporation on the humidity profile was previously 
demonstrated during laboratory experiments by Mestayer et al. (1989).

The measured aerosol concentrations suggest however that this enhancement is only a fraction of the surface layer water vapour content. Integration of the data shown in Fig. 4, for instance, which were measured in gale winds of $25 \mathrm{~m} / \mathrm{s}$, yields a total volume concentration of 2300 $\mu \mathrm{m}^{3} / \mathrm{cm}^{3}$. Since the ambient relative humidity during these measurements was $65 \%$, the total volume when these particles left the sea surface as $3 \%$ salt solutions is approximately 9200 $\mu \mathrm{m}^{3} / \mathrm{cm}^{3}$. Thus the total amount of water vapour released by these particles is estimated as $6.9 \times 10^{-3} \mathrm{~g} / \mathrm{m}^{3}$, or $5.3 \times 10^{-3} \mathrm{~g} / \mathrm{kg}$. This value applies to the mentioned conditions of $25 \mathrm{~m} / \mathrm{s}$ wind speed and $65 \%$ relative humidity, for aerosol observed at $11 \mathrm{~m}$. The correct assessment of the influence of the sea-spray aerosol on the moisture and latent heat budgets in the surface layer requires consideration of the surface-layer flux profiles, rather than concentrations of droplets that are already in equilibrium with ambient relative humidity.

\section{Time-series measurements of particle con- centrations}

In Fig. 8 an example is presented of the time variations of forward scattering intensities and relative humidity. The trace consists of 500 data points, each of these is an average over 20 samples which were recorded with a sampling rate of $200 \mathrm{~Hz}$. The data were taken from the waverider at $2.5 \mathrm{~m}$ above the instantaneous sea surface. A rather periodic pattern is observed in the fluctuations of the particle total area concentrations. The amplitudes of these fluctuations decrease with height above the mean sea surface, but the effects have been observed up to the maximum sampling height of $15 \mathrm{~m}$ as described in De Leeuw (1989b). Visual observations, supported by video recordings, indicate that the fluctuations are correlated with the displacements of the underlying water surface. Spectral analysis of the fluctuation of particle total area concentrations has been presented in De Leeuw (1989b). As an example, we show in Fig. 9 the frequency spectrum of the time series presented in Fig. 8, obtained from an FFT over all 10,000 data

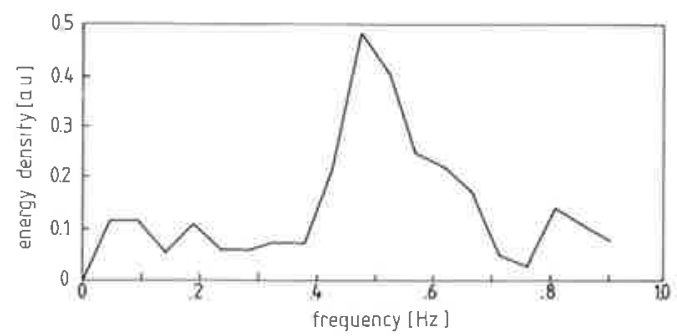

Fig. 9. Fourier spectrum of the optical-scatterometer data presented in Fig. 8.

points. The occurrence of the peak of the spectral density near $0.5 \mathrm{~Hz}$, while wave spectra in the North Sea peak around $0.1 \mathrm{~Hz}$, indicate that it is not the high-energy (low-frequency) waves that cause the observed fluctuations, but rather the somewhat less energetic waves with higher frequencies. This is in agreement with our visual observations. Apparently the aerosol responds quickly enough to follow the low-frequency surface displacements, whereas at higher frequencies the aerosol cannot follow the surface fluctuations. Cross-correlation between the aerosol fluctuations and the waves would yield quantitative insight, but unfortunately the wave measurements and the aerosol measurements were separated by about $25 \mathrm{~m}$. This renders any attempt to correlate the results meaningless. New experiments, including concurrent measurements of the wave parameters and the aerosol are required to yield quantitative information on the cross-correlation and the phase relation between the aerosol fluctuations and the waves.

\section{Time-resolved measurements of relative humidity}

As reported in De Leeuw (1989b), waveinduced effects on the relative humidity were not observed during our time-series measurements. Also an obvious dependence on higher frequencies was not revealed by the spectral analyses, in contrast to the expected behaviour. Probably this is due to the slow response of the hygrometer $(1 \mathrm{~Hz})$, which cannot follow high-frequency turbulent fluctuations. On the other hand, the response time of $1 \mathrm{~s}$ of this instrument is fast enough to indicate the slow fluctuations due to 
surface displacements, as observed for the aerosol. The absence of any wave influence in the humidity spectra, combined with the observed wave-induced particle concentration fluctuations, indicates that the time scale for transport of water vapour by turbulent diffusion is much faster than the time scale that applies to particle transport.

\section{Summary and conclusions}

Data were reported from measurements in the marine atmospheric surface layer of particle size distributions, particle total area concentrations and relative humidity. Profiles were recorded, both of the mean quantities and of time-dependent fluctuations. The particle measurements were made with two devices that are based on different physical principles. The results are in good agreement. The optical scatterometer was especially constructed for the HEXMAX experiment with two applications in mind. In the first place an instrument was required for quick profiling of the surface layer in support of the Rotorod measurements. The observed waveinduced fluctuations of the aerosol total area concentrations made it necessary to apply relatively long averaging times to obtain reliable mean values. Nevertheless, the method is useful because the time-consuming processing, involving digitizing and digital processing of microscope images of the Rotorod samples is not necessary. With the proper calibration, the output voltage of the optical scatterometer yields the aerosol total area concentration in real time. Therefore the optical scatterometer is a useful instrument that supplements the Rotorod. The advantage of the latter is the size information in the profiles.

The second application of the optical scatterometer is the measurement of time-dependent aerosol concentration fluctuations near the airsea interface. The results reveal the influence of the waves on the particles, which is expected to extend above the highest altitude where we measured $(15 \mathrm{~m})$. The particle concentration fluctuations are correlated with the surface displacements. Spectral analyses show that the fluctuations at the dominant frequencies are related to the surface displacements, rather than to the

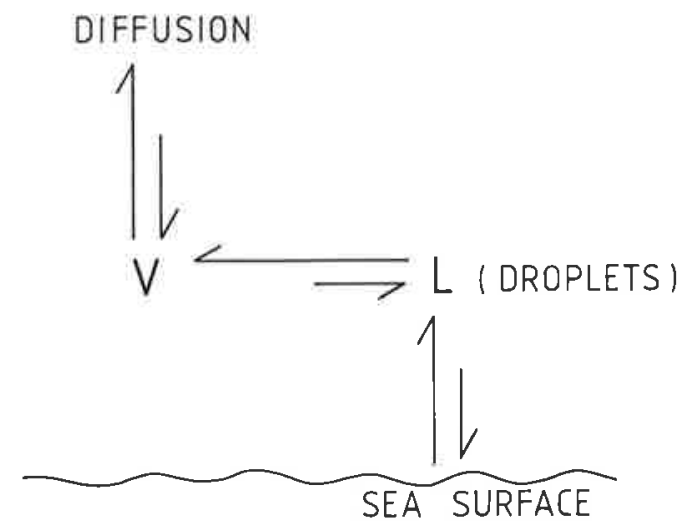

Fig. 10. The dynamic equilibrium between liquid water (L) and vapour (V) shifts due to the faster removal of vapour from the surface layer.

energy in the waves. As a result, the particle fluctuation spectra are peaked at higher frequencies than the wave spectra.

The optical scatterometer and the hygrometer were used for simultaneous recording of particle total area concentration profiles and humidity profiles. The similarities between these profiles indicate the strong interaction between the seaspray droplets and the humidity field.

The humidity spectra are not correlated with the surface displacements. This indicates that the time scales for the transport of water vapour are much faster than those for particle transport. Due to the faster removal of vapour which follows the air flow, as opposed to the greater droplets that lag behind due to their larger inertia, the equilibrium between liquid water and vapour is disturbed (cf. Fig. 10). This is because the relative humidity decreases when water vapour is removed and therefore the evaporation losses cannot be balanced by condensation on the falling particles. This leads to the conclusion that net liquid water transport takes place from sea to air. Since the droplets lose mass due to evaporation as they are transported away from the surface, this means that the liquid water flux decreases with height in the surface layer.

The results are also of importance for modeling of the production and dispersal of aerosol particles near the air-sea interface. A theoretical approach has been outlined in De Leeuw (1989b), which may be used in obtaining information from the experimental data on the contributions 
of various transport mechanisms, such as turbulence and the previously proposed waverotor model. The application of this approach may also yield information on flow separation in oceanic conditions, which is important for wave generation models.

\section{Acknowledgements}

The author appreciates comments by Dr. E. C. Monahan and by Drs C. W. Lamberts. Particular credit is due to Dr. W. A. Oost (KNMI), Dr. K. B. Katsaros (Univ. Washington) and Dr. S. D. Smith (BIO) for perfectly organizing the HEXMAX experiment. The optical scatterometer has been developed and constructed in a cooperative effort of different research sections of the Physics Group of the Physics and Electronics Laboratory TNO. The instrument was assembled by M. Deutekom, electronic circuits were designed by Ing. L. H. Cohen, software for data collection and processing was prepared by Ir. G. J. Kunz who also made the initial laboratory tests. Further support was given by Drs J. A. Boden, Drs C. W. Lamberts, Ir. A. J. T. de Krijger and M. M. Moerman. The hygrometer was prepared by J. Verwey. Software for the analysis was prepared by Ing. B. J. Neger. The mast and waverider buoy were constructed by $\mathrm{L}$. Bruinsma and P. W. van Kuivenhoven. Meteorological data were obtained from Meetpost Noordwijk, owned by Rijkswaterstaat (the Dutch Department of Harbors and Public Works). The measurements by the Physics and Electronics Laboratory TNO were made in the framework of assignment A86KM103 from the Royal Netherlands Navy, with support from TNO "beleidsruimte subsidie". This article was finished while the author held a National Research CouncilNaval Postgraduate School (Monterey, CA, USA) research associateship. It is HEXOS publication 13.

\section{REFERENCES}

Anisimova, Ye. P., Speranskaya, A. A. and Likhacheva, O. N. 1976. Deformation of the wind velocity profile over developing wind waves. Izv. Atmos. Oceanic Phys. 12, 748-754.

Banner, M. L. and Melville, W. K. 1976. On the separation of air flow over water waves. $J$. Fluid Mech. 77, 825-842.

Blanchard, D. C. 1983. The production, distribution, and bacterial entichment of the sea-salt aerosol. In: Air-sea exchange of gases and particles (eds. P. S. Liss and W. G. N. Slinn). Reidel, Dordrecht, Holland, 407-454.

Bortkovskii, R. S. 1987. Air-sea exchange of heat and moisture during storms. Reidel, Dordrecht, Holland.

De Leeuw, G. 1986a. Vertical profiles of giant particles close above the sea surface. Tellus 38B, 51-61.

De Leeuw, G. 1986b. Size distributions of giant aerosol particles close above sea level. J. Aerosol. Sci. 17, 293-296.

De Leeuw, G. 1987. Near-surface particle size distribution profiles over the North Sea. J. Geophys. Res. 92, 14631-14635.

De Leeuw, G. 1988. Mixed-layer profiling with lidar and modeling of the aerosol vertical structure. Proc. NATO Adv. Workshop: Humidity exchange over the sea main experiment (HEMAX) analysis and interpretation (eds. W. A. Oost, S. D. Smith and K. B. Katsaros). Tech. Rep. Dept. Atm. Sci., AK-40, Univ. Washington, Seattle, WA 98195, USA, pp. 100-104.
De Leeuw, G. 1989a. Modeling of extinction and backscatter profiles in the marine mixed-layer. Appl. Opt. 28, 1356-1359.

De Leeuw, G. 1989b. Investigations on turbulent fluctuations of particle concentrations and relative humidity in the marine atmospheric surface layer. J. Geophys. Res. 94, 3261-3269.

Fairall, C. W., Davidson, K. L. and Schacher, G. E. 1983. An analysis of the surface production of sea-salt aerosols. Tellus 35B, 31-39.

Fairall, C. W., Davidson, K. L. and Schacher, G. E. 1984. Application of a mixed-layer model to aerosols in the marine boundary layer. Tellus 36B, 203-211.

Fairall, C. W. and Davidson, K. L. 1986. Dynamics and modeling of aerosols in the marine atmospheric boundary layer. In: Oceanic Whitecaps (eds. E. C. Monahan and G. MacNiocaill). Dordrecht, D. Reidel, pp. 195-208.

Hsu, C.-T., Hsu, E. Y. and Street, R. L. 1981. On the structure of turbulent flow over a progressive water wave: Theory and experiment in a transformed, wave-following co-ordinate system. J. Fluid Mech. $105,87-117$.

Katsaros, K. B., Smith, S. D. and Oost, W. A. 1987. HEXOS-Humidity exchange over the sea. A program for research on water-vapor and droplet fluxes from sea to air at moderate to high wind speeds. Bull. Am. Meteor. Soc. 68, 466-476.

Koga, M. and Toba, Y. 1981. Droplet distribution and dispersion processes on breaking wind waves. Sci. 
Rep. Tohoku Univ., Ser. 5 (Tohoku Geophys. J.) 28, $1-25$.

Konishi, T. 1981. Analysis of wind structure over water waves using a flow visualization method. Pap. Meteorol. Geophys. 32, 99-108.

Mestayer, P. G. and Lefauconnier, C. 1988. Spray droplet generation, transport, and evaporation in a wind wave tunnel during the humidity exchange over the sea experiments in the simulation tunnel. J. Geophys. Res. 93, 572-586.

Mestayer, P. G., Edson, J., Fairall, C. W., Larsen, S. E. and Spiel, D. E. 1989. Turbulent transport and evaporation of droplets generated at an air-water interface. In: Turbulent Shear Flows, Vol. 6 (eds. J.-C. Andre et al.). Berlin, Springer-Verlag, pp. 129-147.

Monahan, E. C. 1988. Whitecap coverage as a remotely monitorable indication of the rate of bubble injection into the oceanic mixed layer. In: Sea Surface Sound (ed. B. R. Kerman). Kluwer Academic Publ. pp. 85-96.

Monahan, E. C., Spiel, D. E. and Davidson, K. L. 1986. A model of marine aerosol generation via whitecaps and wave disruption. In: Oceanic Whitecaps (eds. E. C. Monahan and G. MacNiocaill). Dordrecht, D. Reidel, pp. 167-174.

Wills, J. A. B. 1984. HEXOS Model tests on the Noordwijk Tower. Report R184, British Maritime Technology, Teddington, Middlesex TW11 0JJ, UK (53 pp.).

Wu, J., Murray, J. J. and Lai, R. J. 1984. Production and distributions of sea spray. J. Geophys. Res. 89, 8163-8169. 